\title{
Performance characterization of mid- infrared difference frequency generation in orientation-patterned gallium phosphide
}

Junxiong Wei, Chaitanya Kumar S., Hanyu Ye, Peter G. Schunemann, M. Ebrahim-Zadeh

Junxiong Wei, Chaitanya Kumar S., Hanyu Ye, Peter G. Schunemann, M. Ebrahim-Zadeh, "Performance characterization of mid-infrared difference frequency generation in orientation-patterned gallium phosphide," Proc. SPIE 10516, Nonlinear Frequency Generation and Conversion: Materials and Devices XVII, 105160V (15 February 2018); doi: 10.1117/12.2287670

SPIE. Event: SPIE LASE, 2018, San Francisco, California, United States 


\title{
Performance characterization of mid-infrared difference frequency generation in orientation-patterned gallium phosphide
}

\author{
Junxiong Wei*a, S. Chaitanya Kumar ${ }^{\mathrm{a}, \mathrm{b}}$, Hanyu Ye ${ }^{\mathrm{a}}$, Peter G Schunemann ${ }^{\mathrm{c}}$, and \\ M. Ebrahim-Zadeh ${ }^{\text {a,b,d }}$ \\ ${ }^{a}$ ICFO-Institut de Ciencies Fotoniques, The Barcelona Institute of Science and Technology, 08860 \\ Castelldefels (Barcelona), Spain; ${ }^{b}$ Radiantis, Polígon Camí Ral, 08850 Gavà, Barcelona, Spain; \\ ${ }^{c}$ BAE Systems Incorporated, MER15-1813, P.O. Box 868, Nashua, New Hampshire 03061-0868,

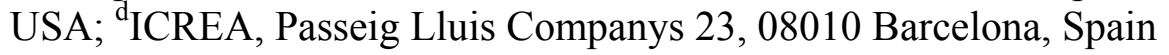

\begin{abstract}
Orientation-patterned gallium phosphide (OP-GaP) is a recently developed nonlinear material with wide transparency across $0.8-12 \mu \mathrm{m}$ and high nonlinearity $\left(d_{14} \sim 70 \mathrm{pm} / \mathrm{V}\right)$, which is a promising candidate material for mid-infrared generation. Here we report the full performance characterization of a tunable single-pass nanosecond difference frequency generation (DFG) source based on OP-GaP by mixing the output of a Q-switched Nd:YAG laser at $1.064 \mu \mathrm{m}$ with the signal from a pulsed MgO:PPLN OPO pumped by the same laser. Using the longest OP-GaP crystal (40 mm) deployed to date, the DFG source provides up to $\sim 14 \mathrm{~mW}$ of average output power at $2719 \mathrm{~nm}$ at $80 \mathrm{kHz}$ repetition rate, with $>6 \mathrm{~mW}$ across $2492-2782 \mathrm{~nm}$, in $\mathrm{TEM}_{00}$ spatial profile. By performing relevant measurements, detrimental issues such as residual absorption and thermal effects have been studied and confirmed. The temperature and spectral acceptance bandwidths for DFG in the $40-\mathrm{mm}-\log \mathrm{OP}-\mathrm{GaP}$ are measured to be $18{ }^{\circ} \mathrm{C}$ and $17 \mathrm{~nm}$, respectively, at 1766 $\mathrm{nm}$. The DFG beam exhibits passive power stability better than $1.7 \%$ rms over 1.4 hours at $2774 \mathrm{~nm}$, compared to $1.6 \%$ and $0.1 \%$ rms for the signal and pump, respectively. The polarization dependence of the input beams on the DFG power has also been systematically investigated, for the first time to our knowledge. Further, we have measured the damage threshold of the OP-GaP crystal to be $0.8 \mathrm{~J} / \mathrm{cm}^{2}$ at $1064 \mathrm{~nm}$.
\end{abstract}

Keywords: nonlinear optics, difference frequency generation, Orientation-patterned gallium phosphide, acceptance bandwidths, polarization dependence, damage threshold

\section{INTRODUCTION}

The availability of efficient tunable laser sources emitting in the mid-infrared (IR) region of the electromagnetic spectrum from $2 \mu \mathrm{m}$ to $10 \mu \mathrm{m}$ is extremely important for a large variety of applications [1-3]. Numerous efforts have been directed towards the development of such mid-IR sources in this particular wavelength range [4-6] and nonlinear optical sources provide the most effective approach to transfer the favorable characteristics of near-IR solid-state lasers with high efficiency to practically any wavelength with high-power, widely tunable, in the mid-IR region. To date, optical parametric oscillators (OPOs) based on MgO-doped PPLN (MgO:PPLN) are firmly established as the most effective sources to generate tunable high-power radiation in the spectral range of 2-4 $\mu \mathrm{m}$, limited by the onset of multiphonon absorption beyond $\sim 4 \mu \mathrm{m}$ [7]. Hence, nonlinear frequency conversion sources at longer wavelengths are still lacking in performance and simplicity, driving researchers to investigate alternative nonlinear materials, which can provide extended mid-IR spectral coverage. Traditionally, access to this mid-to-deep-IR spectral range is best achieved by nonlinear frequency conversion techniques, such as DFG [8], OPO [9] or cascaded OPOs [10] primarily pumped by the well-established Nd:YAG or Yb-fiber lasers at $1064 \mathrm{~nm}$. At the same time, suitable materials with the required linear and nonlinear optical properties, in addition to the long-wavelength transparency cut-off, are key factors for in the development of practical and efficient frequency conversion sources. Several material parameters, including effective nonlinear coefficient $\left(d_{\mathrm{eff}}\right)$, type of interaction and the polarizations of the incident fields, as well as temperature and spectral phase-matching properties, are critical factors for efficient frequency conversion processes. One of the most recently developed nonlinear optical materials orientation-patterned gallium phosphide (OP-GaP), a QPM semiconductor crystal offering promising linear and nonlinear properties for mid-to-deep-IR generation. It overcomes several limitations of the established nonlinear materials such as $\mathrm{ZnGeP}_{2}$ and $\mathrm{OP}-\mathrm{GaAs}$, which require pumping laser at wavelengths above $\sim 2 \mu \mathrm{m}$ [11]. OP-GaP exhibits higher thermal conductivity, noncritical phase-matching, and a larger band gap, which

Nonlinear Frequency Generation and Conversion: Materials and Devices XVII, edited by

Konstantin L. Vodopyanov, Kenneth L. Schepler, Proc. of SPIE Vol. 10516, 105160V

(c) 2018 SPIE · CCC code: $0277-786 X / 18 / \$ 18 \cdot$ doi: $10.1117 / 12.2287670$ 
allow the generation of mid-to-deep-IR radiation using established pump laser technology at $1 \mu \mathrm{m}[12,13]$. However, during this early stage in the development of this new nonlinear crystal, several growth parameters are yet to be established to achieve the expected performance in frequency down-conversion processes into the mid-to-deep-IR. Hence, it is imperative to perform detailed characterization of the linear and nonlinear optical properties to investigate the viability of this material for frequency conversion in different time-scales.

Earlier reports on frequency conversion in OP-GaP include a nanosecond doubly-resonant OPO (DRO) pumped at 1064 $\mathrm{nm}$, generating $4 \mathrm{~mW}$ of idler at $4624 \mathrm{~nm}$ and $15 \mathrm{~mW}$ of signal at $1324 \mathrm{~nm}$ at $10 \mathrm{kHz}$ [14], as well as a nanosecond DRO pumped at $2090 \mathrm{~nm}$ operating at a fixed idler wavelength of $5100 \mathrm{~nm}$ and a signal wavelength of $3540 \mathrm{~nm}$, providing a total signal plus idler output power of $350 \mathrm{~mW}$ at $20 \mathrm{kHz}$ [15]. Recently, we also demonstrated a tunable DFG source based on OP-GaP operating across $2548-2781 \mathrm{~nm}$ by mixing the input pulses from a nanosecond Nd:YAG pump laser and the signal from a MgO:PPLN OPO in a 40-mm-long crystal, providing up to $\sim 14 \mathrm{~mW}$ of average output power at 80 $\mathrm{kHz}$ repetition rate [16]. In another report, we demonstrated the first optical parametric generator (OPG) based on OPGaP. The OPG, pumped by a Q-switched Nd:YAG laser at $1064 \mathrm{~nm}$, can be temperature tuned across 1721-1850 nm in the signal and $2504-2787 \mathrm{~nm}$ in the idler, providing up to $\sim 18 \mathrm{~mW}$ of the total average output power at $25 \mathrm{kHz}$ repetition rate, with $\sim 5 \mathrm{~mW}$ of the idler [17]. Further, in the picosecond pulse regime, our group also demonstrated a single-pass DFG between the output signal of a picosecond OPO and the input pump pulses in OP-GaP, tunable across 3040-3132 $\mathrm{nm}$, providing up to $57 \mathrm{~mW}$ of DFG power at $80 \mathrm{MHz}$ repetition rate [18]. On the other hand, in the CW regime, singlepass DFG based on 16.5-mm-long OP-GaP crystal was reported, providing up to $150 \mathrm{~mW}$ at $3400 \mathrm{~nm}$ for an input pump power of $47 \mathrm{~W}$ at $1064 \mathrm{~nm}$ together with $24 \mathrm{~W}$ of signal power at $1550 \mathrm{~nm}$ [19]. Recently, using a $24.6 \mathrm{~mm}$-long-OP$\mathrm{GaP}$ crystal, CW DFG power of $65 \mu \mathrm{W}$ was generated at $5850 \mathrm{~nm}$ for a pump power of $10 \mathrm{~W}$ at $1064 \mathrm{~nm}$ and a signal power of $40 \mathrm{~mW}$ at $1301 \mathrm{~nm}$ [20].

Here we report a systematic performance characterization of the single-pass mid-IR DFG in a 40-mm-long OP-GaP crystal, for the first time. Performance characteristics including parasitic wavelength generation, mid-IR DFG tuning, power scaling, temperature as well as spectral acceptance bandwidths are measured. Further, the DFG power dependence on the polarization of the incident fields has also be investigated. The transmission measurements at the pump and signal wavelength together with thermal effects and damage threshold limitation of our OP-GaP sample are presented.

\section{EXPERIMENTAL SETUP}

The schematic of the experimental setup for the single-pass DFG in OP-GaP crystal is similar to that in Ref. [16], as depicted in Fig.1. The primary pump source is a Q-switched Nd:YAG laser (Bright Solutions, Sol), delivering up to 30 $\mathrm{W}$ of average power at $1064.7 \mathrm{~nm}$ in linear polarization, with tunable repetition rate. The output pulse-width varies from $8 \mathrm{~ns}$ at $20 \mathrm{kHz}$ to $26 \mathrm{~ns}$ at $100 \mathrm{kHz}$ repetition rate. However, for the experiments presented in this report, we used the pump laser at $80 \mathrm{kHz}$, resulting in a pump pulse duration of $23 \mathrm{~ns}$. The instantaneous bandwidth and frequency jitter of this laser, measured using an optical spectrum analyzer, are $<0.5 \mathrm{~nm}$ and $\sim 1 \mathrm{~nm}$ over 0.5 minutes, respectively. A major part of the output power from the laser is used to pump a pulsed OPO, which provides the signal beam for the DFG in $\mathrm{OP}-\mathrm{GaP}$, while the remaining output power from the laser is used as the pump for the DFG process. The nonlinear gain medium for the pulsed OPO is a 38-mm-long, 1-mm thick, MgO:PPLN crystal, with five grating periods ranging from $\Lambda_{\mathrm{MgO}: \mathrm{PPLN}}=29.5$ to $31.5 \mu \mathrm{m}$ in steps of $0.5 \mu \mathrm{m}$. However, in the present DFG experiments we have used a single grating period of $\Lambda_{\mathrm{MgO}: \mathrm{PPLN}}=31.5 \mu \mathrm{m}$. The end faces of the crystal are antireflection (AR)-coated for high transmission $(R<4 \%)$ for the pump and idler, high reflection $(R<1 \%)$ for the signal over 1300-1900 nm. The crystal is housed in an oven which can be adjusted from room temperature to $200{ }^{\circ} \mathrm{C}$, with a stability of $\pm 0.1{ }^{\circ} \mathrm{C}$. The OPO is configured as a singly resonant oscillator for the signal to be resonant in a V-cavity. In order to partially extract the signal from the cavity we used a $\sim 22 \%$ output coupler, while the idler is extracted in single-pass. The OPO can be temperature tuned over 1664$1928 \mathrm{~nm}$ in the signal and 2374-2950 $\mathrm{nm}$ in the idler for the fixed grating period of $\Lambda_{\mathrm{MgO}: \mathrm{PPLN}}=31.5 \mu \mathrm{m}$. The signal from the OPO and the remaining pump from the laser are collimated and combined using a dichroic mirror which is ARcoated for high reflection $(R>99 \%)$ for the signal over 1300-2000 nm and high transmission $(T>90 \%)$ for the pump at $1064 \mathrm{~nm}$. The beams are focused to beam waist radius of $w_{\mathrm{op}} \sim 65 \mu \mathrm{m}, w_{\mathrm{os}} \sim 60 \mu \mathrm{m}$ for the pump and signal, respectively, at the center of the OP-GaP crystal for DFG. These focal spots correspond to a focusing parameter of $\xi_{\mathrm{p}} \sim 0.5$ and $\xi_{\mathrm{s}} \sim 1$ for the pump and signal, respectively. The OP-GaP crystal used for DFG is a 40-mm-long and has a physical aperture of $6 \times 1.7 \mathrm{~mm}^{2}$ with a single grating period of $\Lambda_{\mathrm{DFG}}=15.5 \mu \mathrm{m}$. The resulting DFG beam waist in the OP-GaP crystal is estimated to be $w_{\mathrm{ODFG}} \sim 44 \mu \mathrm{m}\left(\xi_{\mathrm{DFG}} \sim 2.6\right)$. The end faces of the crystal are AR-coated for high transmission $(R<1 \%)$ at 
$1064 \mathrm{~nm}$ and $1500-1900 \mathrm{~nm}$, with $>80 \%$ transmission over $2500-2800 \mathrm{~nm}$. The generated DFG is extracted separated from the pump and signal using a Ge filter and measured using a power meter.

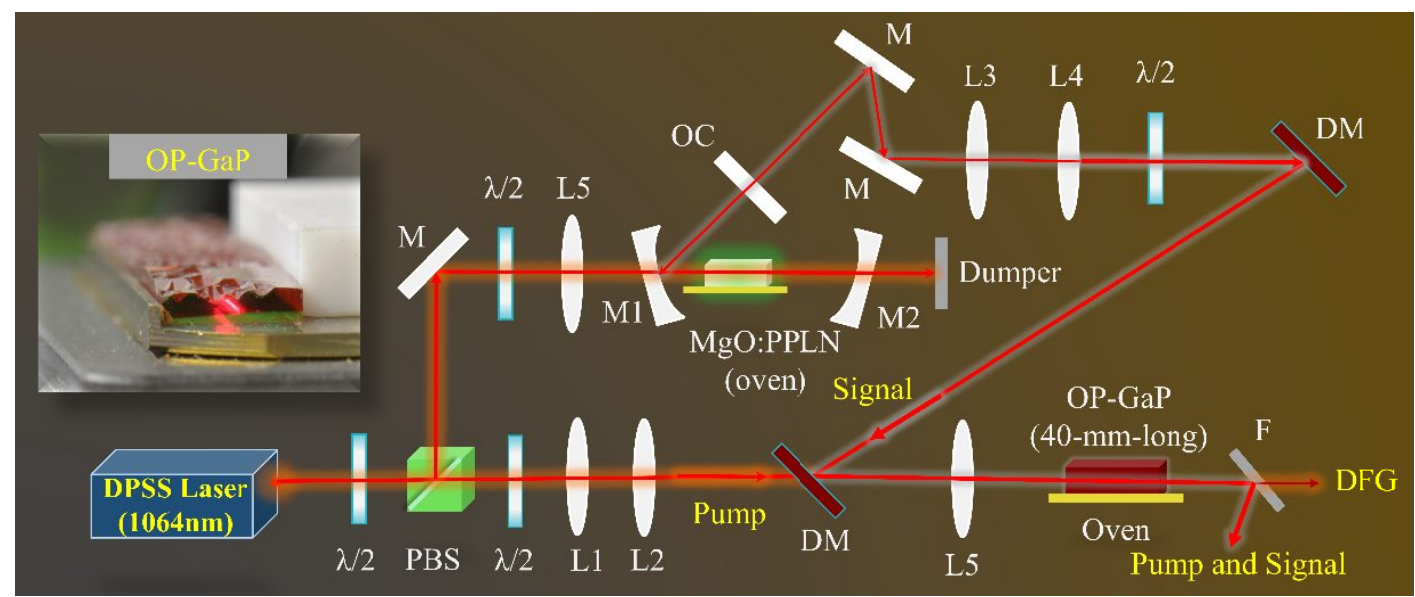

Fig. 1. Schematic of the experimental setup. $\lambda / 2$ : Half-wave-plate, PBS: Polarizing beam-splitter, OC: Output coupler, DM: Dichroic mirror, M: Mirrors, L: Lens, F: Germanium filter. Inset: 40-mm-long OP-GaP crystal generating red parasitic output beam at the exit of the crystal.

\section{EXPERIMENTAL RESULTS}

\subsection{Parasitic processes}

Initially, with the pump beam incident on the sample, we observed a weak green light originating from non-phasematched frequency doubling of the pump light. By optimizing spatial overlap of the pump and signal beams through the OP-GaP sample, a parasitic output beam was observed at the exit of the crystal, as shown in the inset of Fig.1. Figure 2(a) illustrates a typical spectrum of the generated parasitic light at $664.6 \mathrm{~nm}$, measured using a visible spectrometer (Ocean Optics, HR4000), corresponding to higher-order QPM sum frequency generation (SFG) of the $1064 \mathrm{~nm}$ pump and the $1770 \mathrm{~nm}$ signal, at an $\mathrm{OP}-\mathrm{GaP}$ crystal temperature of $115^{\circ} \mathrm{C}$. Occasionally, we have also observed some weak frequency doubling of the signal beam. Further, another parasitic SFG between the DFG and the pump at $761.4 \mathrm{~nm}$ is also generated together with the DFG output. The total power of the parasitic wavelengths was measured to be $<1 \mathrm{~mW}$, compared to the generated DFG output power of $\sim 14 \mathrm{~mW}$.
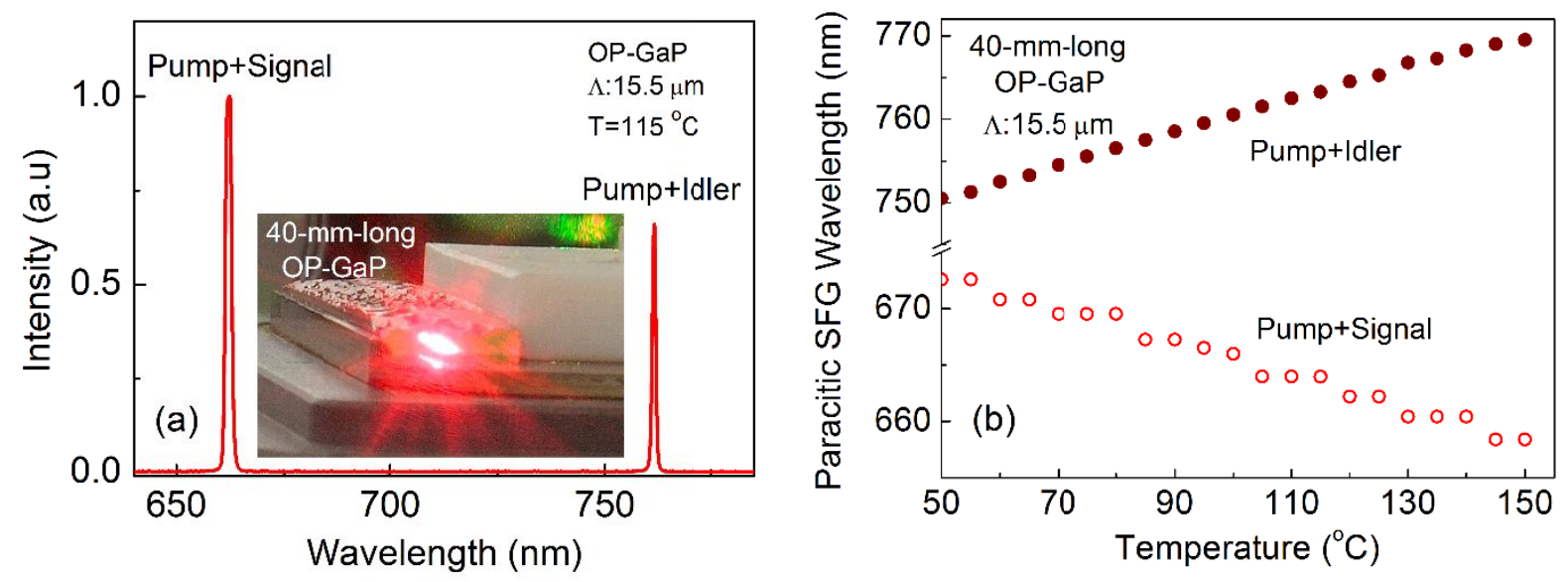

Fig. 2. (a) Spectrum of the generated parasitic SFG at $664.6 \mathrm{~nm}$ (Pump+Signal) and $761.4 \mathrm{~nm}$ (Pump+DFG). Inset: OP-GaP crystal generating parasitic output in the visible. (b) Variation of the parasitic SFG wavelengths as a function of OP-GaP crystal temperature.

In order to characterize the DFG source, we performed spectral tuning of the output by varying the signal wavelength of the OPO, and simultaneously adjusted the phase-matching temperature of the OP-GaP crystal at a fixed pump power of 3 $\mathrm{W}$ and a signal power of $0.6 \mathrm{~W}$. By varying the temperature of the MgO:PPLN crystal from $105^{\circ} \mathrm{C}$ to $170{ }^{\circ} \mathrm{C}$, and the 
temperature of $\mathrm{OP}-\mathrm{GaP}$ from $150{ }^{\circ} \mathrm{C}$ to $50{ }^{\circ} \mathrm{C}$, we were able to achieve tunable parasitic SFG from 658 to $672 \mathrm{~nm}$ (Pump+Signal) and 770-753 nm (Pump+DFG). Hence, the achievable DFG tuning is calculated to be from 2548-2781 $\mathrm{nm}(233 \mathrm{~nm})$ with $>5 \mathrm{~mW}$ of output power across the tuning range.

\subsection{Acceptance bandwidth}

In order to further study the phase-matching properties of the OP-GaP crystal for DFG process, we performed temperature acceptance measurements under the focusing condition used for DFG, with $w_{\mathrm{op}} \sim 65 \mu \mathrm{m}$ and $w_{\mathrm{os}} \sim 60 \mu \mathrm{m}$, corresponding to a focusing parameter of $\xi_{\mathrm{p}} \sim 0.5$ and $\xi_{\mathrm{s}} \sim 1$ for the pump and signal, respectively, at the center of the nonlinear crystal. For this measurement, the pump and signal powers were maintained constant at $3 \mathrm{~W}$ and $0.6 \mathrm{~W}$, respectively. For a DFG wavelength of $2676 \mathrm{~nm}$, the normalized DFG power as a function of the temperature deviation is shown in Fig.3(a), where it can be seen that the $\operatorname{sinc}^{2}$ fit to the experimental data resulted in a full-width at halfmaximum (FWHM) temperature acceptance bandwidth of $\Delta \mathrm{T} \sim 18{ }^{\circ} \mathrm{C}$, centered at $\sim 103{ }^{\circ} \mathrm{C}$. This is much wider than the theoretically estimated bandwidth of $\Delta \mathrm{T}=1{ }^{\circ} \mathrm{C}$ for a 40 -mm-long OP-GaP crystal with a grating period of $\Lambda=15.56 \mu \mathrm{m}$, using the temperature-dependent Sellmeier equations [14], as shown in Fig. 3(b). Further, we investigated the spectral acceptance bandwidth of the 40-mm-long OP-GaP crystal. The normalized DFG power as a function of the signal wavelength for a fixed pump wavelength, is shown in Fig. 3(c). For this measurement, the phase-matching temperature is optimized for every signal wavelength to achieve maximum DFG power. As can be seen in Fig. 3(c), the $\operatorname{sinc}^{2}$ fit to the experimental data results in a FWHM spectral acceptance bandwidth of $\Delta \lambda_{\mathrm{s}} \sim 17 \mathrm{~nm}$. This is also much wider than the theoretically estimated bandwidth of $\Delta \lambda_{\mathrm{s}} \sim 4 \mathrm{~nm}$ for a 40 -mm-long OP-GaP crystal at wavelength of $1760 \mathrm{~nm}$ using the temperature-dependent Sellmeier equations [14], as shown in Fig. 3(d).
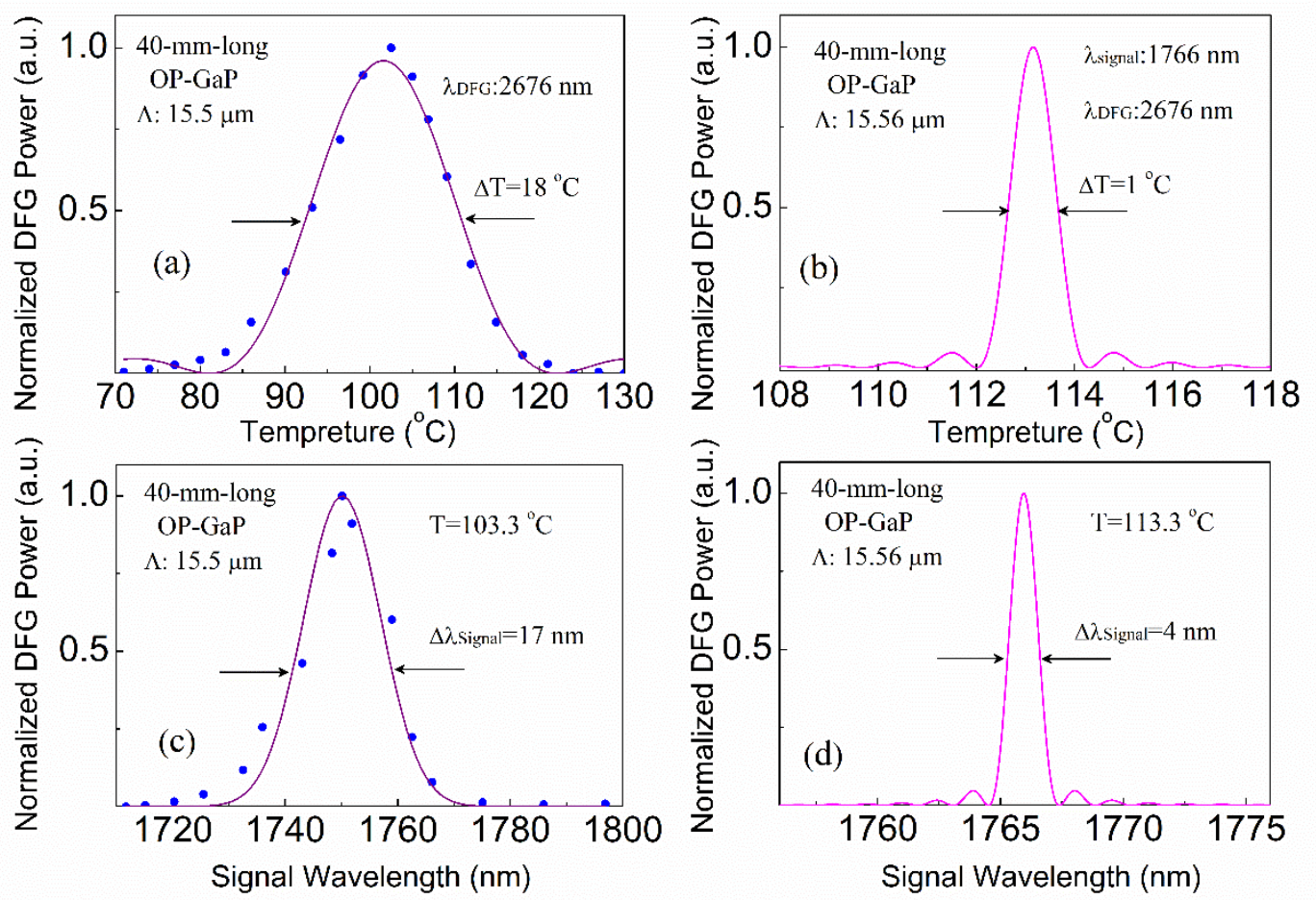

Fig.3. Experimentally measured, and theoretically calculated temperature acceptance bandwidth for the 40-mm-long OP-GaP crystal.

It is also to be noted that the theoretically calculated phase-matching temperature is $\sim 129^{\circ} \mathrm{C}$, which is much higher than the measured experimental value $103.3{ }^{\circ} \mathrm{C}$ for an OP-GaP grating period of $\Lambda=15.5 \mu \mathrm{m}$. The phase-matching temperature estimated for the grating period of $\Lambda=15.56 \mu \mathrm{m}$ is $122{ }^{\circ} \mathrm{C}$, as shown in Fig. 3(d), indicating that the difference in the phase-matching temperature is caused by the uncertainty in the grating period, additional thermal effects, and broad bandwidths of the pump and signal pulses used to in the DFG experiments. 


\subsection{Polarization dependence and optimization}

In order to study the dependence of the frequency conversion on the incident beam polarization in OP-GaP, we measured the DFG power for different pump polarizations. For both incident pump and signal beams linearly polarized, there are three special cases to be considered [19], with the results presented in Fig. 4(a-c). Two independent half-wave plates were used to vary the pump and signal polarizations, and the measured DFG power was normalized with respect to that when the pump beam polarization was along the direction $[01 \overline{1}]$ in the crystal $\left(\psi \mathrm{p}=90^{\circ}\right)$. The corresponding theoretically calculated results showing the variation of the gain factor, $\delta^{2}$, as a function of input signal polarization $(\psi \mathrm{s})$ for three different fixed pump polarizations $(\psi \mathrm{p})$ are presented in the insets of Fig. 4(a-c). For a fixed pump polarization along the [100] direction $\left(\psi \mathrm{p}=0^{\circ}\right)$, and constant pump power of $3 \mathrm{~W}$ at $1064 \mathrm{~nm}$ and signal power of $0.6 \mathrm{~W}$ at $1748 \mathrm{~nm}$, the variation of the normalized DFG power as a function of signal polarization is shown in Fig. 4(a). As expected, the normalized DFG power varies sinusoidally with minimum at $\psi \mathrm{s}=0^{\circ}$, reaching a maximum of $\sim 1.2$ for signal polarization perpendicular to the pump polarization direction $\left(\psi \mathrm{s}=90^{\circ}\right)$. Similar measurements for orthogonal pump polarization of $\psi \mathrm{p}=90^{\circ}$ resulted in a constant output for all directions of signal polarization, as shown in Fig. 4(b). Further, when the pump polarization is fixed at $\psi \mathrm{p} \sim 54.7^{\circ}$, the normalized DFG power again varies sinusoidally, reaching a maximum of $\sim 1.4$ for a signal polarization angle of $\psi \mathrm{s} \sim 54.7^{\circ}$, as evident from Fig. 4(c). This value is close to the theoretically expected value of 1.3 .
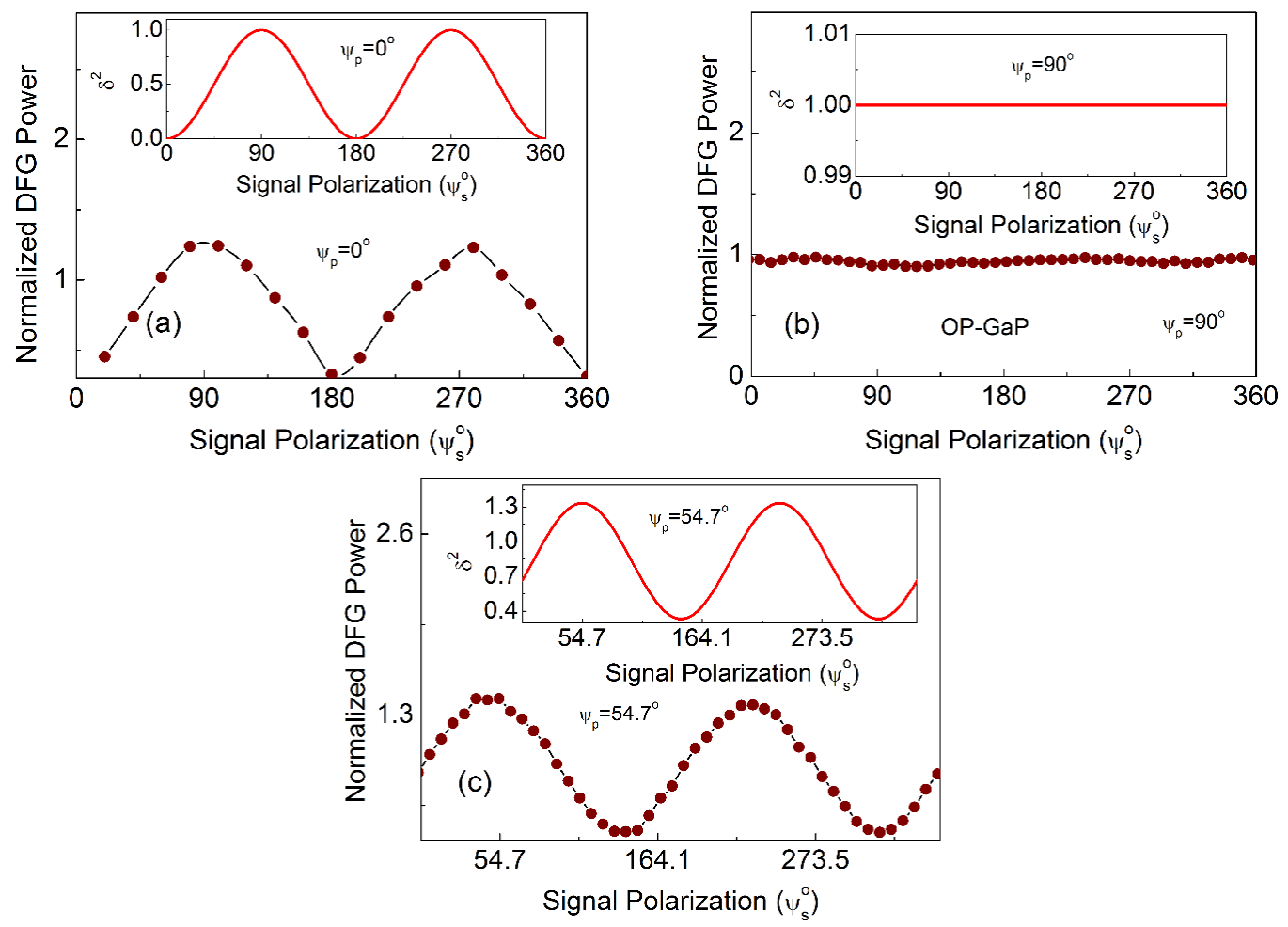

Fig. 4. Variation of the normalized DFG power as a function of signal polarization, $\psi \mathrm{s}$, for a fixed pump polarization of (a) $\psi \mathrm{p}=0^{\circ}$, (b) $\psi \mathrm{p}=90^{\circ}$, (c) $\psi \mathrm{p}=54.7^{\circ}$. Inset: Corresponding theoretical calculations.

\subsection{Power scaling}

We also performed power-scaling measurements of our DFG source based on OP-GaP. The variation of the DFG output power as a function of the input pump power at a pump polarization angle of $\psi \mathrm{p} \sim 54.7^{\circ}$ is shown in Fig. 5. For a fixed signal power of $1 \mathrm{~W}$ at $1748 \mathrm{~nm}$, and changing the pump power at the input to the OP-GaP crystal up to $5 \mathrm{~W}$, we were able to generate a maximum of $13.8 \mathrm{~mW}$ at $2719 \mathrm{~nm}$. The DFG power is observed to increase linearly with a slope efficiency of $0.3 \%$, as shown in Fig. 5(a). For each pump polarization, the signal polarization was optimized to achieve maximum DFG power. Further, we also measured the transmission of the OP-GaP crystal for the input beams at the same position where we generated maximum DFG power. The transmission for the pump and signal beams were 
recorded to be $28 \%$ and $18 \%$, respectively, while generating the DFG. Considering the losses in the crystal, the generated maximum DFG power represents a pump to DFG conversion efficiency of $\sim 1 \%$, corresponding to a photon conversion efficiency of $2.5 \%$. A similar measurement for a fixed pump power of $5 \mathrm{~W}$ and varying the signal power is shown in Fig.5(b), where the DFG power is observed to increase linearly with a slope efficiency of $1.2 \%$, generating a maximum DFG power of $13.8 \mathrm{~mW}$ at $1 \mathrm{~W}$ of signal power. It is to be noted that the DFG powers presented here are not corrected for the losses in the crystal and the separating filter. While performing the power scaling measurements, the temperature of the OP-GaP crystal was adjusted to achieve the maximum DFG power. As the pump power was increased from the $0.6 \mathrm{~W}$ to $5 \mathrm{~W}$, the phase-matching temperature of the $\mathrm{OP}-\mathrm{GaP}$ had to be reduced by $\sim 30{ }^{\circ} \mathrm{C}$, indicating strong thermal effects. This change in temperature is much wider that the measured temperature acceptance bandwidth presented in Fig. 3 , and can be attributed to the absorption at the pump and signal wavelengths, resulting in the temperature rise of the OP$\mathrm{GaP}$, thereby necessitating a reduction in the phase-matching temperature. We also recorded the single-pass DFG power obtained across the tuning range of 2713-2793 nm, with the results shown in Fig. 5(c). For each signal wavelength, we optimized the temperature of OP-GaP crystal to obtain maximum DFG power. The source could provide up to $10.2 \mathrm{~mW}$ of mid-IR DFG output at $2774 \mathrm{~nm}$ for $3 \mathrm{~W}$ of pump and $0.6 \mathrm{~W}$ of signal power, with $>6 \mathrm{~mW}$ over the entire tuning range. The inset of Fig.5(c) shows the far-field energy distribution of the DFG output beam at $2690 \mathrm{~nm}$, at maximum power, recorded using a pyroelectric camera. The result indicates $\mathrm{TEM}_{00}$ mode profile with a single-peak Gaussian distribution. Similar beam profiles were observed across the DFG tuning range.
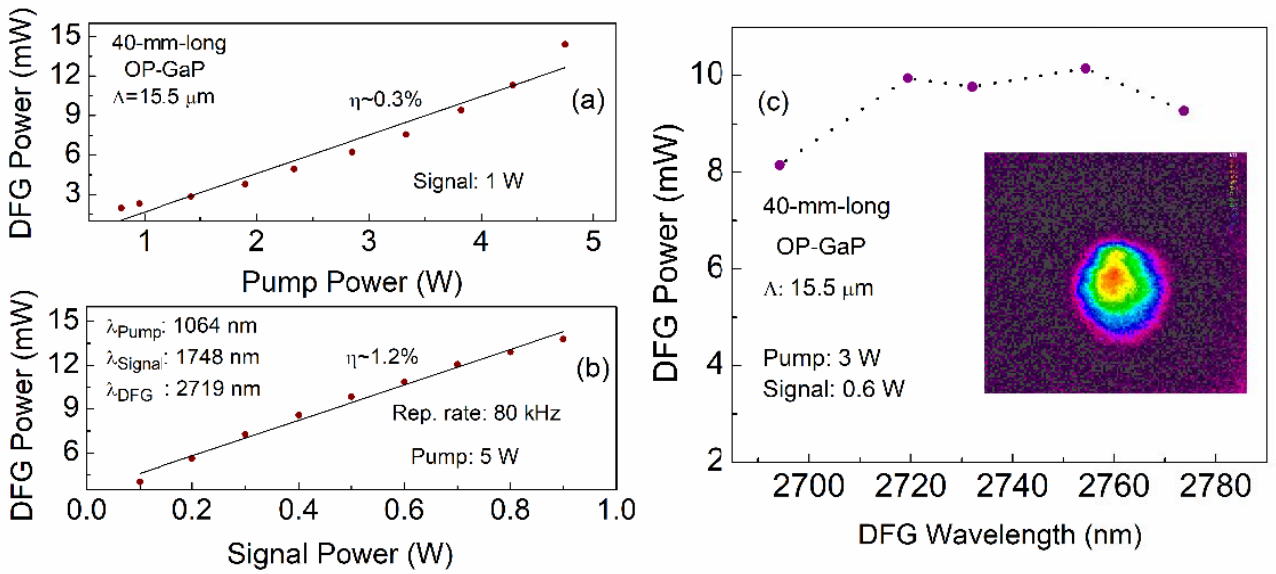

Fig. 5. Dependence of the measured DFG power at $2719 \mathrm{~nm}$ on (a) incident pump power for fixed OPO signal power, and (b) incident OPO signal power for fixed pump power. (c) DFG power across the tuning range. Inset: Spatial beam profile of the DFG output beam.

A similar measurement for a fixed pump power of $5 \mathrm{~W}$ resulted in a maximum DFG power $14 \mathrm{~mW}$ at a slope efficiency of $1.2 \%$ with respect to the signal power. It is to be noted that the DFG powers presented here are not corrected for the losses in the crystal and the separating filters used during the experiment. Also shown in the inset of Fig. 5 is the spatial beam profile of the DFG beam recorded using mid-IR beam profiler, while generating maximum power. Further, the passive power stability of the DFG power is recorded to be better than $1.7 \%$ rms over 1.4 hours at $2774 \mathrm{~nm}$.

\subsection{Thermal effects and damage Limitations}

The low transmission at the pump and signal wavelengths immediately suggests the onset of thermal effects in the OP$\mathrm{GaP}$ crystal. Hence, we performed systematic measurements to estimate the temperature rise on the surface of the nonlinear crystal using a point contact thermocouple. As the pump power at the input to the OP-GaP crystal was increased to a maximum of $5 \mathrm{~W}$, with no signal input, a temperature of $\sim 17^{\circ} \mathrm{C}$ was measured. Similarly, when the incident signal power was increased to $1 \mathrm{~W}$, with no input pump, the temperature rise was recorded to be only $\sim 4.5^{\circ} \mathrm{C}$. On the other hand, while generating the maximum DFG power of $14 \mathrm{~mW}$, with $5 \mathrm{~W}$ of pump and $1 \mathrm{~W}$ signal power at the input to the OP-GaP crystal, the temperature on the surface of the crystal increased by $\sim 20^{\circ} \mathrm{C}$. Further, we observed that the time required to reach the maximum DFG power is longer for an incident pump power of $5 \mathrm{~W}$ with fixed signal power, than that required when the pump power is fixed with an incident signal power of $1 \mathrm{~W}$. When the signal power is fixed, the crystal reaches a steady state temperature and releasing the pump power leads to a large temperature rise. Hence, it requires a longer time to reach the final steady-state phase-matching temperature. These measurements indicate that the absorption at the pump wavelength is much more significant than at the signal and DFG wavelengths. Such high 
absorption at the pump wavelength could eventually lead to damage in the OP-GaP crystal. Hence, we also attempted to characterize our OP-GaP crystal for damage at the $1064 \mathrm{~nm}$. The pump beam was focused to a waist radius of $w_{\mathrm{op}} \sim 75$ $\mu \mathrm{m}$ at the center of the crystal. The maximum tolerable average power before the crystal experience damage increased linearly from $1.8 \mathrm{~W}$ at $20 \mathrm{kHz}$ to $12 \mathrm{~W}$ at $80 \mathrm{kHz}$. It is to be noted that the pulse duration also varies from $8 \mathrm{~ns}$ at $20 \mathrm{kHz}$ to $23 \mathrm{~ns}$ at $80 \mathrm{kHz}$, which is accounted for in the calculated peak-intensity, resulting in a damage threshold of $\sim 0.84$ $\mathrm{J} / \mathrm{cm}^{2}$. Owing to low transmission at the pump wavelength, this damage threshold is $\sim 6.4$ times lower than that reported in the literature for $\mathrm{GaP}[23]$.

\section{CONCLUSIONS}

In conclusion, we have reported a systematic study on the performance characteristics of a tunable nanosecond DFG source based on the new semiconductor nonlinear material, OP-GaP. The source has been realized by single-pass DFG between a Q-switched nanosecond laser at $1064 \mathrm{~nm}$ the signal beam from a pulsed OPO based on MgO:PPLN pumped by the same laser. The DFG source can provide up to $\sim 14 \mathrm{~mW}$ of average output power at $2719 \mathrm{~nm}$ at $80 \mathrm{kHz}$ repetition rate, with $>6 \mathrm{~mW}$ across the full tuning range, in $\mathrm{TEM}_{00}$ spatial profile. The spectral and temperature acceptance bandwidths for the DFG process in OP-GaP have been measured to be $18{ }^{\circ} \mathrm{C}$ and $17 \mathrm{~nm}$, respectively. The corresponding theoretical calculations indicate some discrepancy due to the grating period. We have also performed detailed characterization on the dependence of gain in OP-GaP for DFG on the polarization of incident pump and signal fields. These resulted in the achievement of maximum DFG power when the incident beams are polarized along the body diagonal, in good agreement with the theory. Moreover, thermal effects were studied by recording the increase in the surface temperature of our OP-GaP crystal. By exploiting the repetition rate tunability of our pump laser, we have also estimated the damage threshold of OP-GaP to be $\sim 0.84 \mathrm{~J} / \mathrm{cm}^{2}$. To the best of our knowledge, this is the first report on complete characterization of the OP-GaP crystal for pulsed DFG. We believe that with further progress in the growth of OP-GaP crystal with higher transmission, better QPM grating quality, substantial improvements in the efficiency, output power, and beam quality will be attainable.

\section{Funding}

We acknowledge support from Ministerio de Economía y Competitividad (MINECO) (nuOPO, TEC2015-68234-R); European Commission (Mid-Tech, H2020-MSCA-ITN-2014); European Office of Aerospace Research and Development (EOARD) (FA9550-14-1-0390); Agència de Gestió d'Ajuts Universitaris i de Recerca (AGAUR) (SGR 2014-2016); Severo Ochoa (SEV-2015-0522); Fundación Cellex

\section{REFERENCES}

[1] M. W. Sigrist, "Mid-infrared laser-spectroscopic sensing of chemical species," J. Adv. Res. 6(3), 529-533 (2015).

[2] V. A. Serebryakov, É. V. Bơ̌ko, N. N. Petrishchev, and A. V. Yan, "Medical applications of mid-IR lasers. Problems and prospects," J. Opt. Technol. 77(1), 6-17 (2010).

[3] M. Ebrahim-Zadeh and I. T. Sorokina, Mid-Infrared Coherent Sources and Applications, New York, NY, USA: Springer, 2008.

[4] M. Ebrahim-Zadeh and K. Vodopyanov, "Mid-infrared coherent sources and applications: introduction," J. Opt. Soc. Am. B 33(11), MIC1-MIC1 (2016).

[5] S. D. Jackson, " Towards high-power mid-infrared emission from a fibre laser," Nat. Photonics 6(7), 423-431 (2012).

[6] Y. Yao, A.J. Hoffman, and C. Gmachl, "Mid-infrared quantum cascade lasers," Nat. Photonics 6(7), 432-439 (2012).

[7] R. Bhatt, I. Bhaumik, S. Ganesamoorthy, R. Bright, M. Soharab , A. K. Karnal , and P. K. Gupta, " Control of intrinsic defects in lithium niobate single crystals for optoelectronic applications," Crystals 7(2), 23 (2017).

[8] K. Devi, P. G. Schunemann, and M. Ebrahim-Zadeh, "Continuous-wave, multimilliwatt, mid-infrared source tunable across 6.4-7.5 $\mu \mathrm{m}$ based on orientation-patterned GaAs," Opt. Lett. 39(23), 6751-6754 (2014).

[9] R. K. Feaver, R. D. Peterson, and P. E. Powers, "Longwave-IR optical parametric oscillator in orientationpatterned GaAs pumped by a $2 \mu \mathrm{m}$ Tm,Ho:YLF laser," Opt. Express 21(13), 16104-16110 (2013). 
[10] M. Henriksson, M. Tiihonen, V. Pasiskevicius, and F. Laurell, "Mid-infrared ZGP OPO pumped by neardegenerate narrowband type-I PPKTP parametric oscillator," Appl. Phys. B 88(1), 37-41 (2007).

[11] V. Petrov, "Parametric down-conversion devices: The coverage of the mid-infrared spectral range by solid-state laser sources," Opt. Mater. 34(3), 536-554 (2012).

[12]V. Petrov, "Frequency down-conversion of solid-state laser sources to the mid-infrared spectral range using non-oxide nonlinear crystals," Prog. Quantum Electron. 42, 1-106 (2015).

[13] P. G. Schunemann, K. T. Zawilski, L. A. Pomeranz, D. J. Creeden, and P. A. Budni, "Advances in nonlinear optical crystals for mid-infrared coherent sources,” J. Opt. Soc. Am. B 33(11), D36-D43 (2016).

[14] L. A. Pomeranz, P. G. Schunemann, D. J. Magarrell, J. C. McCarthy, K. T. Zawilski, and D. E. Zelmon, "1- $\mu$ mpumped OPO based on orientation-patterned GaP," Proc. SPIE 9347, 93470K (2015).

[15]P. G. Schunemann, L. A. Pomeranz, and D. J. Magarrell, "First OPO based on orientation-patterned gallium phosphide (OP-GaP)," in CLEO: Science and Innovations (Optical Society of America, 2015), paper SW3O-1.

[16] J. Wei, S. Chaitanya Kumar, H. Ye, K. Devi, P. G. Schunemann, and M. Ebrahim-Zadeh, Nanosecond difference frequency generation in orientation-patterned gallium phosphide. Opt. Lett. 42(11), 2193-2196 (2017).

[17] H. Ye, S. Chaitanya Kumar, J. Wei, P. G. Schunemann, M. Ebrahim-Zadeh, "Optical parametric generation in orientation-patterned gallium phosphide," Opt. Lett. 42(18), 3694-3697 (2017).

[18] J. C. Casals, S. Parsa, S. C. Kumar, K. Devi, P. G. Schunemann, and M. Ebrahim-Zadeh, "Picosecond difference-frequency-generation in orientation-patterned gallium phosphide," Opt. Express 25(16), 1959519602 (2017).

[19] S. Guha, J. O. Barnes, and P. G. Schunemann, "Mid-wave infrared generation by difference frequency mixing of continuous wave lasers in orientation-patterned Gallium Phosphide," Opt. Mater. Express 5(12), 2911-2923 (2015).

[20] G. Insero, C. Clivati, D. D’Ambrosio, P. De Natale, G. Santambrogio, P. G. Schunemann, J. J. Zondy, and S. Borri, "Difference frequency generation in the mid-infrared with orientation-patterned gallium phosphide crystals," Opt. Lett. 41(21), 5114-5117 (2016).

[21] M. R. Lorenz, G. D. Pettit, and R. C. Taylor, "Band gap of gallium phosphide from 0 to $900^{\circ} \mathrm{K}$ and light emission from diodes at high temperatures," Phys. Rev. 171(3), 876-881(1968).

[22] A. L. Bajor "Investigation of stress-induced birefringence in large semiconductor wafers by imaging polarimetry," Proc. SPIE 2265, 431-442 (1994).

[23] Y. Li, F. Liu, Y. Li, L. Chai, Q. Xing, M. Hu and C. Wang, "Experimental study on GaP surface damage threshold induced by a high repetition rate femtosecond laser," Appl. Opt. 50(13), 1958-1962 (2011). J. F. Van Derlofske, "Computer modeling of LED light pipe systems for uniform display illumination," Proc. SPIE 4445, 119-129 (2001). 